\title{
Philosophiques
}

\section{Les conditions de la sécession}

\section{Allen Buchanan}

Volume 19, numéro 2, automne 1992

Une nation peut-elle se donner la constitution de son choix?

URI : https://id.erudit.org/iderudit/027197ar

DOI : https://doi.org/10.7202/027197ar

Aller au sommaire du numéro

Éditeur(s)

Société de philosophie du Québec

ISSN

0316-2923 (imprimé)

1492-1391 (numérique)

Découvrir la revue

Citer ce document

Buchanan, A. (1992). Les conditions de la sécession. Philosophiques, 19(2),

159-168. https://doi.org/10.7202/027197ar d'utilisation que vous pouvez consulter en ligne.

https://apropos.erudit.org/fr/usagers/politique-dutilisation/ 


\title{
LES CONDITIONS DE LA SÉCESSION ${ }^{\mathrm{I}}$
}

\author{
par
}

\section{Allen Buchanan}

Est-il possible - ou plutôt, est-il permis - à une nation de se donner la constitution de son choix? Si par « nation », on entend tout peuple ou groupe ethnique, c'est-à-dire tout groupe possédant une langue, une histoire et une culture communes, et si « se donner la constitution de son choix » signifie devenir un Etat complètement indépendant et souverain, alors je crois que la réponse à cette question est non.

On dit parfois que toute nation dans ce sens large (tout peuple ou groupe ethniquel a le droit à l'autodétermination. Cette position est défendable, et peut-être même non problématique, mais uniquement si le droit à l'autodétermination attribué à chaque «nation » dans ce sens large n'inclut pas le droit de toute «nation » à posséder son propre État souverain.

Le fait de posséder un État souverain n'est qu'une forme, la plus extrême des formes, de l'autodétermination pour un groupe. Donc même s'il est vrai que toute nation entendue dans le sens large, c'est-à-dire tout peuple ou groupe ethnique, a le droit à l'autodètermination, il ne s'ensuit pas que toute nation, toujours dans le même sens du terme « nation », a le droit à un Etat souverain.

Le principe selon lequel tout peuple ou groupe ethnique doit avoir (et a le droit d'avoir) son propre État souverain est parfois appelé le principe normatif nationaliste. Ce principe suppose que

1. Ce texte a éte traduit par Geneviève Sicotte. 
toute nation (entendue au sens large) a le droit de se séparer de tout État qu'elle ne contrôle pas exclusivement.

Le principe normatif nationaliste devrait être rejeté, et ce pour plusieurs raisons. La première, comme je viens de le suggérer, est que ce principe repose sur le présupposé erroné voulant que le droit à l'autodétermination implique un droit à la souveraineté (et donc un droit à la sécession). De plus, le principe selon lequel toute « nation » dans le sens large, c'est-à-dire tout peuple ou groupe ethnique, doit avoir son propre État souverain, nie catégoriquement la légitimité de tout État abritant plus d'un groupe ethnique, du moins si l'un de ces groupes aspire à la souveraineté. Il s'agit là d'une conséquence très troublante, puisque le pluralisme ethnique est souvent considéré comme l'un des traits distinctifs de l'État moderne, ou à tout le moins de l'Etat moderne libéral.

De plus, le nombre de groupes ethniques ou de peuples n'est pas limité, mais pourrait augmenter par l'immigration ou autrement; cest dire que le principe normatif nationaliste, qui assimile le droit à l'autodétermination au droit à la souveraineté et donne à tout peuple ou groupe ethnique le droit à l'autodétermination, pourrait donner lieu à une fragmentation politique illimitée.

Mais ce n'est pas tout. Même sans tenir compte de l'instabilité et des coûts èconomiques de la fragmentation illimitée qu'il sanctionne, le principe normatif nationaliste suscite une objection beaucoup plus sérieuse. Ernest Gellner formule la difficulté comme ceci:

Pour parler simplement: il $y$ a un grand nombre de nations potentielles sur terre. Notre planete contient aussi de la place pour un certain nombre d'entités politiques indépendantes ou autonomes. Mais n'importe quel calcul raisonnable montre que le nombre des premières (les nations potentielles) excède sans doute très, très largement le nombre d'Etats possibles. Si cet argument ou ce calcul est correct, tous les nationalismes ne peuvent pas être satisfaits, et surtout pas tous au même moment. La satisfaction des uns fait la frustration des autres. Ce qui vient appuyer et renforcer encore cet argument, c'est le fait qu'un très grand nombre des nations potentielles de ce monde vivent ou ont vécu jusqu'à récemment non pas dans des entitès territoriales circonscrites, mais mêlées les unes aux autres selon des structures complexes. Dans de tels cas, il s'ensuit qu'une entite territoriale politique ne peut devenir ethniquement homogène que si elle tue, expulse ou assimile tous les habitants $n^{\prime}$ appartenant pas à son ethnie dominante?

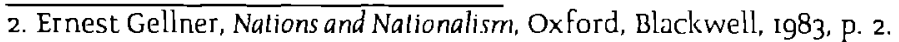


Ainsi, il n'est pas suffisant de soutenir que l'on doit rejeter le principe selon lequel chaque nation, c'est-à-dire chaque peuple ou groupe ethnique, doit avoir son propre État souverain, sous prétexte que c'est un principe irréalisable ou trop coûteux économiquement. Ce principe doit être rejeté parce que même une simple tentative de le reconnaitre entraînerait des coûts moraux inacceptables.

On peut maintenant voir pour quelles raisons on doit répondre par la négative à notre question de départ, si une «nation » signifie simplement tout peuple ou groupe ethnique, et $\mathrm{si}$ « se donner la constitution de son choix » suppose que la nation se sépare de l'État qui l'abrite et qu'elle devient souveraine. Les « nations » prises dans ce sens large n'ont pas le droit à la souveraineté. Elles peuvent avoir le droit à l'autodétermination, mais le droit à l'autodétermination n'inclut pas le droit à la souveraineté, et donc à la sécession.

Mais peut-être n'est-ce pas là le sens qu'il faut donner au terme « nation » tel qu'il apparaît dans notre question. $\mathrm{Si}$, au lieu de l'interprétation proposée, «nation » signifie ici un groupe ayant droit à son propre Etat souverain, alors la réponse à notre question est oui. Car évidemment, si un peuple a droit à la souveraineté, il a aussi le droit de déterminer la forme constitutionnelle de cette souveraineté - c'est-à-dire « le droit de se donner la constitution de son choix ». Mais si c'est ainsi que l'on doit comprendre notre question, la réponse, bien qu'affirmative, n'a rien de bien excitant. En fait, il s'agit d'une véritable tautologie, et au surplus pas très intéressante. Tout dépendra alors de savoir quels sont les groupes qui constituent des nations en ce sens spécifique, c'est-à-dire quels sont les groupes ayant droit à la souveraineté et donc ayant le droit de faire sécession s'ils se retrouvent dans un Etat qu'ils ne contrôlent pas exclusivement.

Même s'il est vrai qu'une « nation » dans ce second sens (c'est-à-dire un groupe ayant droit à la souveraineté) peut se donner la constitution de son choix, il ne s'ensuit pas que le Québec puisse le faire, à moins que les Québécois ne constituent véritablement une nation dans ce second sens, un groupe ayant droit à son propre Etat souverain. Mais c'est précisément ce que certains remettraient en question. Jen conclus que la réponse à la question « une nation peut-elle se donner la constitution de son choix? » est soit trivialement vraie, soit non trivialement fausse, et que dans aucun de ces cas elle ne jette une quelconque 
lumière sur la question de savoir si oui ou non le Québec a le droit de faire sécession.

Il est plus éclairant de se concentrer sur les deux questions suivantes. Premièrement, le Québec at-il le droit de faire sécession - et si oui, de quelle sorte de droit s'agit-il? Deuxièmement, nonobstant le fait que le Québec ait ou non ce droit, quelles devraient être les conditions justes de cette sécession? En ce qui concerne la seconde question, celle des conditions justes de la sécession, je m'arrêterai en particulier à deux aspects cruciaux et émotivement chargès: la question territoriale et le problème du statut des autochtones du Québec.

Lorsqu'on parle de droit à la sécession, on peut vouloir parler soit d'un droit moral, soit d'un droit légal. Un droit légal à la sécession devrait être soit un droit constitutionnel, soit un droit reconnu par le droit international. Quelques Etats ont inclu dans leur constitution le droit à la sécession - en particulier l'Union Soviétique, la fédération de Malaisie (pendant un court laps de temps), et plus récemment la Tchécoslovaquie. Le Canada ne figure toutefois pas parmi ces Etats. Le Québec n'a pas de droit constitutionnel à la sécession.

On ne peut pas non plus soutenir de façon convaincante que le Québec a un droit à la sécession en vertu du droit international. Le droit international concernant le droit à la sécession est un fouillis scandaleux. Le plus que l'on puisse dire, c'est qu'en pratique, le droit international a toujours reconnu un droit à la sécession uniquement aux colonies du tiers monde soumises à une domination étrangère.

Au niveau des principes, le droit international se trouve dans une situation problématique à cause de son adhésion à deux principes incompatibles, et qui en plus sont tous deux indéfendables en eux-mêmes. Le premier est le principe d'inviolabilité de l'intégrité territoriale de tous les Etats existants. Ce principe est indéfendable principalement parce qu'il néglige le fait que des États existants ont obtenu des parties de leur territoire par l'agression pure et simple. Le second principe est celui selon lequel tous les peuples ont le droit à l'autodétermination, par laquelle ils peuvent librement déterminer leur statut politique. Ce principe, si on le prend au pied de la lettre et de façon inconditionnelle, commet l'erreur de confondre l'autodétermination avec la souveraineté, et est sujet à toutes les objections formulées plus haut. Mais comme je viens de le faire remarquer, en pratique, le principe du droit à l'autodétermination de tous les 
peuples n'a jamais été appliqué de façon inconditionnelle. Son application a plutôt été limitée aux cas clairs de décolonisation.

Ces deux principes se révèlent inconciliables parce que le principe d'inviolabilité de l'intégrité territoriale des États existants interdit toute sécession pour quelque raison que ce soit, tandis que le principe du droit à l'autodétermination politique de tous les peuples (à moins qu'il ne soit pondéré) sanctionne une fragmentation virtuellement illimitée des États existants. Le plus que l'on puisse dire pour rendre ces principes conciliables est que le principe d'inviolabilité de l'intégrité territoriale doit être compris comme ne s'appliquant pas aux Etats comprenant des peuples assujettis, c'est-à-dire des groupes ethniques subissant l'oppression active d'autres groupes contrôlant l'État. Cette restriction convient au cas des peuples colonisés, mais pas à celui du Québec actuel. Par conséquent, même s'il est possible de donner une cohérence au droit international en ce qui concerne le droit à la sécession - ce qui est loin d'être sûr - il ne viendrait toujours pas étayer la revendication du Québec à un droit légal à la sécession.

Mais au-delà du droit légal, qu'en est-il du droit moral à la sécession? Dire qu'un groupe possède un droit moral à la sécession, c'est dire au moins deux choses: premièrement, que même en l'absence d'un droit légal, il est moralement acceptable pour un groupe de faire sécession s'il le souhaite; deuxièmement, que même en l'absence d'une obligation légale, les autres parties impliquées ont une très forte obligation morale de ne pas intervenir dans la sécession du groupe.

La sécession ne consiste pas uniquement dans la rupture des liens politiques: elle implique aussi la prise d'un territoire. Une justification solide pour la sécession devrait donc comporter, de la part des sécessionnistes, une revendication valable sur le territoire concerné.

Une grande partie de mon récent ouvrage, Secession: The Morality of Political Divorce ${ }^{3}$, tente de préciser les raisons pouvant justifier qu'un groupe ait un droit moral à la sécession. Parmi ce que je considère être de bonnes justifications pour un droit à la sécession, la seule qui me semblait pouvoir être applicable au cas du Québec était ce quej'appelle « l'argument pour la préservation culturelle ». Selon cet argument, un groupe peut faire sécession

3. Allen Buchanan, Secession: The Morality of Political Divorce From Fort Sumter to Lithuania and Quebec, Boulder, Colorado, Westview Press, Ig9o. 
si cela est le seul moyen de protéger sa culture. Dans le livre, je soulevais quelques questions difficiles quant à savoir si cet argument s'appliquait au cas du Québec, et j'énumérais certaines conditions qui devraient être satisfaites si l'on voulait que cet argument justifie le droit à la sécession. Depuis ce temps, j'ai acquis la conviction que plusieurs sécessionnistes québécois, et peut-être la plupart, veulent la sécession non pas pour protéger leur culture de la destruction, mais pour pouvoir librement déterminer dans quel sens se fera le développement futur de cette culture.

Cette position me semble comporter une difficulté. La position selon laquelle le besoin de protéger une culture de la destruction engendre le droit à la sécession est beaucoup plus solide et défendable que la position voulant que tout groupe désirant avoir le plein contrôle sur le développement de sa culture ait le droit à la sécession. Le problème avec la seconde position est qu'elle semble accorder beaucoup trop. En fait, si un tel droit était gènéralement reconnu et appliqué, la situation serait tout aussi chaotique et moralement intolérable que celle qui résulterait de l'adhésion au principe voulant que chaque peuple ou groupe ethnique ait le droit à la souveraineté. En d'autres termes, ce qui m'apparaît maintenant constituer l'argument principal pour appuyer le droit du Québec à la sécession me semble souffrir des mêmes défauts que le principe normatif nationaliste.

Évidemment, je ne soutiens pas que le besoin de déterminer le sens du développement de sa culture ne fournit pas à un groupe un motif pour revendiquer un certain droit à l'autodétermination. Je me demande simplement si le besoin de contrôler le développement d'une culture fournit un motif pour accéder à la plus radicale des formes d'autodétermination, c'est-à-dire à la sécession entrainant la formation d'un État souverain.

Il est temps de résumer le résultat de ma discussion jusqu'à présent. Le Québec n'a pas de droit légal à la sécession, ni selon la constitution, ni selon le droit international. On ne peut pas non plus soutenir que le besoin de déterminer le sens de développement de sa culture ou le droit à l'autodétermination lui donnent un droit moral à la sécession.

On peut toutefois arguer que le fait que le gouvernement canadien ait reconnu le référendum de ig8o sur la souverainetéassociation, ainsi que le fait que le public en général ait considéré ce référendum comme légitime, montrent non 
seulement que la plupart des Canadiens croient que le Québec a le droit de faire sécession, mais qu'ils accordent de ce fait un droit à la sécession. Je crois que cet argument possède un certain poids, mais il se trouve en quelque sorte affaibli à cause de la grande confusion qui régnait sur ce qu'était précisément la souveraineté-association, et donc sur le type de droit qui était reconnu ou accordé.

Ceci dit, je voudrais insister sur le fait qu'en fin de compte, je crois qu'il n'est pas très important de savoir si oui ou non le Québec a le droit de faire sécession. S'il y a dans la province une claire majorité en faveur de la sécession, le Canada doit alors accepter que le processus de sécession soit mis en marche. Cest toutefois une chose que de dire qu'il est juste de permettre la sécession, et c'en est une autre que de déterminer les conditions de cette sécession. Je traiterai donc maintenant de cette question très délicate des conditions de la sécession.

Comme je l'ai noté plus haut, la sécession ne consiste pas uniquement dans la rupture des liens politiques: elle implique aussi la prise d'un territoire. Pour savoir si la sécession est justifiée, et à quelles conditions elle l'est, il faudra ainsi savoir si les sécessionnistes peuvent validement prétendre au territoire qu'ils cherchent à soustraire à l'Etat. La question territoriale ne peut pas être évitée.

De nombeux sécessionnistes québécois présument non seulement que la sécession est justifiable, mais qu'il est évident que les frontières actuelles de la province de Québec seront les frontières d'un Québec indépendant ${ }^{4}$. Ceux qui s'opposent à la sécession du Québec - ou qui l'accepteraient seulement à des conditions peu attrayantes pour les Québécois - invoquent deux raisons pour réfuter cette supposition. Premièrement, disent-ils, environ les deux tiers de la partie nord du Québec ont été cédés par le Canada à la province de Québec, une entité qui fait partie de la féderation canadienne. Deuxièmement, ils affirment que si les Québécois ont droit à la souveraineté, les autochtones y ont aussi droit. (Si les francophones québécois forment une societé, une culture ou un peuple distincts, cest aussi le cas pour les Inuit et les Cris.) La conclusion qu'ils tirent de cela est que si les Québécois ont le droit de faire sécession, ce droit ne

4. Ce qui suit est en partie tiré de mon essai « Quebec Secession and Native Territorial Rights ", Le Réseau (publiè par le Réseau sur la Constitution), vol. 2, $\pi^{*}$ 3, mars 1992, pp. 2-4, et est utilisé avec la permission des éditeurs du Réseau. 
sapplique pas à la province ou au territoire entier, mais seulement à la petite portion où s'établirent à l'origine les colons français. On conclut aussi parfois de ce raisonnement que si les Québècois ont le droit d'avoir leur propre territoire souverain, les autochtones ont aussi ce droit.

Aucun de ces arguments n'est fondé. Malheureusement, de nombreux partisans de la sécession du Québec, pressés de rêfuter ces conclusions, invoquent des arguments tout aussi chancelants qui discréditent à leur insu leur propre cause.

La réplique type des sécessionnistes consiste en deux arguments. Premièrement, en vertu de la Convention de la Baie James et du Nord québécois (I975), tous les droits des autochtones sur le territoire québécois ont été abolis; il ne peut donc pas y avoir de sécession des autochtones. Deuxièmement, il ne peut pas être question de réduire le territoire du Québec puisque selon la Constitution, les frontières d'une province ne peuvent être changées sans son consentement.

Ce second argument est facile à réfuter. La Constitution interdit de changer les frontières de la province de Québec sans son consentement, et la province de Québec est une unité de la fédération canadienne. Cette disposition ne sapplique donc qu'à la condition que le Québec demeure une province.

Le recours à la Convention de la Baie James et du Nord québécois soulève des problèmes plus complexes. La portée véritable de la Convention est sujette à bien des débats. Il est vrai qu'elle abolit les droits des autochtones relativement à la possession du territoire. Toutefois, les autochtones estiment, de façon assez vraisemblable, que la Convention leur garantit un rôle dans la prise de décisions concernant le développement de la région. Ils croient que ce rôle dans la prise de décision sur des questions de contrôle et de compétence est un élément important de leur autodétermination, et qu'ils l'ont obtenu en échange de leur renonciation à toute revendication sur le territoire lui-même.

La signification exacte de la Convention n'est pas essentielle. Ce qui importe, c'est le fait que c'est un accord ayant été conclu dans le cadre de la Constitution fédérale, et que celle-ci donne au gouvernement fédéral le mandat de protéger les intérêts fondamentaux des peuples autochtones. Par conséquent, on ne peut pas présumer que les autochtones auraient accepté les conditions de la Convention si celle-ci n'avait pas été signée dans le contexte de l'obligation du gouvernement fédéral de protéger leurs intérêts, de même que dans le cadre complexe de la législation et de 
l'administration fédérales qui a été développé au cours des decennies pour permettre au gouvernement de s'acquitter de ce mandat de protection. Il s'ensuit donc que l'existence de l'obligation féderale et du cadre institutionnel pour l'exécuter sont une condition de validité pour la Convention. C'est par conséquent une erreur de supposer que la sécession du Québec n'aurait aucune incidence sur la Convention. C'en serait également une de dire que la Convention règle la question territoriale ou le statut des peuples autochtones.

Jestime que les peuples autochtones se montreraient imprudents et le gouvernement fédéral irresponsable s'ils n'exigeaient pas comme condition préalable à la sécession la renégociation du statut des autochtones. On ne peut pas prédire l'issue de ce processus complexe. Toutefois, il faut éviter de s'enfermer dans l'idée préconçue et erronnée selon laquelle les seuls choix qui s'offrent aux autochtones sont soit d'être une simple «minorité culturelle » dans un Québec indépendant, soit de posséder leur propre État souverain. Les sécessionnistes admettront eux-mêmes que la première option est inacceptable. (Après tout, ce sont eux qui se plaignent que les concepts éculés du bilinguisme et du multiculturalisme ne peuvent pas assurer l'autodétermination réelle d'une société distincte.) On ne doit pas croire non plus que la pleine souveraineté est possible ou souhaitable pour les peuples autochtones du Québec. Dans l'économie mondiale actuelle, où seules les entités politiques de grande taille peuvent espérer survivre, le statut d'État indépendant pour une très petite population est un obstacle à une réelle autodétermination.

Toutefois, l'argument antisécessionniste selon lequel les Québécois, dans le cas d'une sécession, n'auraient droit qu'à la petite portion du territoire originellement occupée par leurs ancêtres, est aussi trop simpliste. Il est vrai que la plus grande partie du territoire a été cédée à la province en tant que partie du Canada et en supposant qu'elle demeurerait de même. Néanmoins, le fait que le Québec ait toujours constitué une entité juridique distincte au sein du Canada et qu'il ait eu un statut de plus en plus autonome et spécifique dans la fédération crée certainement une présomption voulant que si la sécession survient, ce sera la sécession du territoire entier. Cependant, cette présomption pourra être relativisée selon que des ajustements seront nécessaires concernant le territoire ou les compétences gouvernementales pour protéger les intérêts légitimes des peuples autochtones, dont leur droit à l'autodétermination. 
Je concluerai en résumant mes arguments principaux. Il importe peu de savoir si le Québec a un droit moral ou légal à la sécession. Il semble y avoir - et je crois qu'il devrait y avoir - un consensus sur le fait que le Québec peut faire sécession si une majorité sans équivoque de Québécois le désire. La question essentielle est alors de savoir quelles sont les conditions justes de la sécession. Parmi les conditions les plus importantes devant assurer que le processus de sécession soit juste, il y a la clarification du statut des peuples autochtones et la protection de leurs intérêts légitimes, incluant leur droit à l'autodétermination.

Il n'appartient pas à d'autres de déterminer à l'avance ce qui devra être accordé pour satisfaire le droit légitime des autochtones à l'autodétermination; cela ne peut émerger qu'à travers un processus équitable dans lequel les autochtones seront autorisés à défendre eux-mêmes leurs intérêts, tels qu'ils les conçoivent. On ne peut pas présumer que la protection adéquate des intérêts des peuples autochtones ne demandera pas qu'on leur accorde des compétences significatives sur une portion du territoire, ou même que l'on procède à des modifications des frontières entre le Canada et le Québec dans le cas où les peuples autochtones choisiraient de demeurer dans le cadre canadien. Cela signifie que la question autochtone et la question territoriale sont inextricablement liées. Aucune ne peut être évitée, et aucune ne peut être résolue sans que l'autre ne doive l'être aussi. Il n'existe pas de voie aisée dans la tâche difficile de déterminer les conditions justes de la sécession.

Département de philosophie

Université d'Arizona (Tucson) 